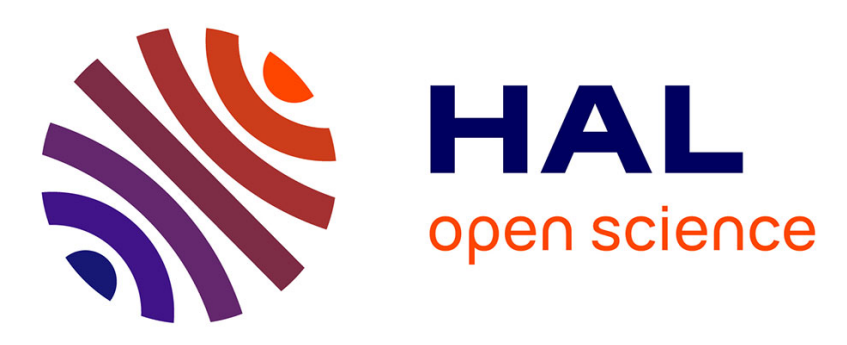

\title{
Nanoelectrode-based devices for electrical biodetection in liquid solution
}

Laurent Malaquin, Christophe Vieu, M Geneviève, Yannick Y. Tauran, Franck

Carcenac, Marie Laure L Pourciel-Gouzy, Véronique Le Berre, Emmanuelle Trévisiol

\section{To cite this version:}

Laurent Malaquin, Christophe Vieu, M Geneviève, Yannick Y. Tauran, Franck Carcenac, et al.. Nanoelectrode-based devices for electrical biodetection in liquid solution. Microelectronic Engineering, 2004, 73-74, pp.887 - 892. 10.1016/j.mee.2004.03.070 . hal-01850070

\section{HAL Id: hal-01850070 https://hal.science/hal-01850070}

Submitted on 5 Jun 2019

HAL is a multi-disciplinary open access archive for the deposit and dissemination of scientific research documents, whether they are published or not. The documents may come from teaching and research institutions in France or abroad, or from public or private research centers.
L'archive ouverte pluridisciplinaire HAL, est destinée au dépôt et à la diffusion de documents scientifiques de niveau recherche, publiés ou non, émanant des établissements d'enseignement et de recherche français ou étrangers, des laboratoires publics ou privés. 


\title{
Nanoelectrode-based devices for electrical biodetection in liquid solution
}

\author{
L. Malaquin ${ }^{\text {a,* }}{ }^{\text {, C. Vieu }}{ }^{\text {a }}$, M. Geneviève ${ }^{\text {a }}$, Y. Tauran ${ }^{\text {a }}$, F. Carcenac ${ }^{\text {a }}$, \\ M.L. Pourciel ${ }^{\mathrm{a}}$, V. Leberre ${ }^{\mathrm{b}}$, E. Trévisiol ${ }^{\mathrm{b}}$ \\ a LAAS-CNRS, 7, avenue du Colonel Roche, 31077 Toulouse, Cedex 4, France \\ ${ }^{\mathrm{b}}$ Genomic Platform of TOULOUSE INSAIDGBA 135, av. de Rangueil, 31077 Toulouse, Cedex 4, France
}

Available online 17 April 2004

\begin{abstract}
The fabrication of nanosystems for the electrical detection of biomolecular interactions is reported. Using a planar process on silicon involving high-resolution electron beam lithography and lift-off, we have demonstrated the reliable fabrication of nanoelectrode-based devices with controlled gap dimensions ranging from $100 \mathrm{~nm}$ down to $20 \mathrm{~nm}$. These devices were used to achieve the electrical detection of gold colloids. We show that binding events can occur in interelectrode gaps, leading to a drastic modification of the electrical transport through the nanojunction. Using a similar approach, electrical measurements in solution were performed by developing Poly(dimetylsiloxane) molded microtanks. First experiments involving gold colloids in solution have shown the possibility of detecting individual nanoparticles. The versatility and the very high sensitivity of these devices added to the simplicity of implementation make this approach very attractive for the fabrication of efficient nanobiosensors.
\end{abstract}

(c) 2004 Elsevier B.V. All rights reserved.

Keywords: Nanoelectrode; Nanoparticle; Biosensor; Electrical addressing; Microfluidics

\section{Introduction}

The recent progress in nanofabrication permits manipulation and immobilization of cells or biomolecules onto solid surfaces. The next challenge is to fabricate efficient nanosystems for exchanging different types of signals with these small bio-entities. The nature of this signal can be mechanical, for example by using microfabricated

\footnotetext{
${ }^{*}$ Corresponding author.

E-mail address: lmalaqui@laas.fr (L. Malaquin).
}

cantilevers [1] that change their resonance frequency under biomolecule fixation onto their surfaces or optical, for example by using minute variation of permittivity that can be used to detect the formation of molecular complexes through surface plasmon resonance [2]. Another route is to use electrical signals for detecting specific reactions between biomolecules. In the latter case, the role of the nanosystem is to convert a biological information contained in the affinity between two molecular species into a measurable electrical signal. The main challenge is to develop detection 
schemes and high sensitive devices, which are compatible with the addressing of a few biomolecules in order to avoid target amplification systems that require complex procedures and which are time consuming.

The approach reported here is based on the fabrication of planar nanoelectrodes on an oxidized silicon substrate exhibiting gaps whose dimensions can be chosen to match the dimension of the entities to be detected. From the point of view of device dimension and sensitivity, this work is intermediary between the detection at a singlemolecule level by measuring the intrinsic conductivity of the biomolecule [3], and the detection involving microelectrodes, colloid labelling and localized metallization of the gap between the microelectrodes [4]. We combine high sensitivity through the use of nanoscale devices that can detect a small number of molecules, with robustness and signal amplification using colloidal labelling. Moreover, we introduce the possibility to achieve the detection in a liquid environment where biomolecules can better adopt the spatial conformation that is crucial for their recognition abilities.

The detection principle is based on the modification of the junction between two electrodes after molecule immobilization, which induces a measurable change in the conductance of the device. We first demonstrate how very high-resolution electron beam lithography (EBL) can be used to reproducibly fabricate sub-10 $\mathrm{nm}$ planar nanoelectrodes onto silicon substrates. Several approaches are investigated in order to connect single or multiple molecules. In particular, parallel addressing of molecules has been achieved using interdigitated multi-electrodes devices compatible with molecular complexes down to $40 \mathrm{~nm}$. Even if we can produce nanoelectrodes exhibiting sub-10 $\mathrm{nm}$ gaps that are well adapted for the ultimate trapping of single biomolecules [3], our main objective is to fabricate a biosensor delivering a reliable electrical signal. As, the conductivity of major biomolecules once fixed onto a surface and dried is very poor [5], we consider that we cannot rely on measuring the intrinsic conductivity of the molecule to detect biomolecular reactions. The use of colloidal labelling thus appears as an elegant procedure for enhancing conductivity changes occurring when the labelled biomolecule bridges the gap between nanoelectrodes. We show that our nanodevices are sensitive to a single colloid particle, demonstrating the possibility of an ultimate detection limit close to the single-molecule level.

\section{Experimental}

Nanoelectrode-based devices were realized using a combination of photolithography and high resolution Electron Beam Lithography on a scanning transmission microscope working at $200 \mathrm{kV}$. We used a planar process on $4 \times 4 \mathrm{~mm}^{2} \mathrm{Si}$ samples covered with a $300 \mathrm{~nm}$ thick thermally grown oxide film. As our electron beam system was not compatible with the fabrication of large patterns, pads and connecting structures were realized using UV photolithography and lift-off of a $5 \mathrm{~nm} \mathrm{Ti} / 15$ $\mathrm{nm}$ Au bilayer deposited by evaporation. We use a conventional PMMA resist layer for e-beam lithography (molecular weight: $996 \mathrm{k}, 150 \mathrm{~nm}$ thick), but due to the very high density of the patterns we want to produce (40 $\mathrm{nm}$ line/space), the development is achieved in pure Iso-Propyl Alcohol (IPA) assisted with ultrasonic agitation. The dissolution rate at room temperature of correctly exposed PMMA is around $10 \mathrm{~nm} / \mathrm{s}$. After development, a thin film of Gold was deposited by sputtering without any metallic adhesion layer. Lift-off was performed using ultrasonic agitation in an acetone bath. Samples were placed under Nitrogen flow before measurements and surface treatments.

Several architectures of electrodes were investigated. In order to obtain a collective response of nanoentities deposited on a surface, we have implemented the fabrication of multi-electrode devices with gap dimensions ranging from $200 \mathrm{~nm}$ down to $30 \mathrm{~nm}$. Typical SEM images of a $25 \mathrm{in}-$ terdigitated nanoelectrode array and a three electrode based device are presented in Fig. 1(a) and (b). These structures were obtained after deposition of a $15 \mathrm{~nm}$ gold film, and lift-off in acetone with ultrasonic agitation for $10 \mathrm{~min}$.

All electrical measurements presented in this paper were performed using a prober equipment, at room temperature with a constant flow of $\mathrm{N}_{2}$. 

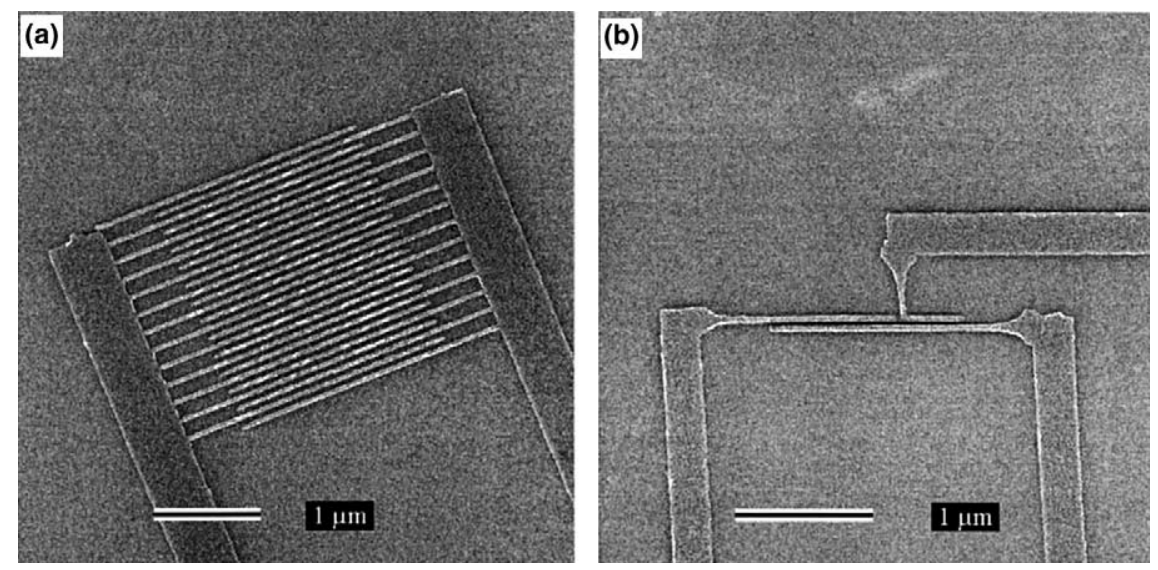

Fig. 1. (a) SEM image of a $40 \mathrm{~nm}$ line and space array of 25 interdigitated nanoelectrodes fabricated by High Resolution EBL and Lift-Off $(20 \mathrm{~nm} \mathrm{Au})$. The total length of the nanojunction of $40 \mathrm{~nm}$ gap is greater than $50 \mu \mathrm{m}$ with no short-cuts. (b) SEM image of a 3electrodes device with $20 \mathrm{~nm}$ gaps obtained by High Resolution EBL and Lift-Off (20 nm Au).

$I(V)$ acquisitions were obtained with a Keithley 4200 semiconductor characterization system giving a typical noise level of $10 \mathrm{fA}$.

Two different schemes were developed in order to perform electrical measurements in solution. They are both based on the fabrication of microchambers deposited on the surface of our devices in order to reduce the volume of reagents, to isolate electrical probes from solution and to avoid evaporation. For the fabrication of these device we used a very well known material, poly(dimethylsiloxane) (PDMS) (Sylgard 184, Dow Corning). This material has been widely studied in particular in the field of rapid prototyping for microfluidic applications [6]. The principle of fabrication of PDMS microchambers by moulding between two stainless steel masters is shown in Fig. 2. PDMS was poured in between the two pieces and cured for $1 \mathrm{~h}$ at $100{ }^{\circ} \mathrm{C}$. Demoulding was performed without any surface treatment of the masters. PDMS chambers were then deposited on the surface of our devices. Reversible sealing was performed simply using the very good conformability capabilities of PDMS on smooth surfaces. This simple and rapid process offers a liquid-tight seal, which is useful for microfluidic application as it is demountable for cleaning or observations. Depending on the structure of the microchamber, the injection of the solution was achieved by using a micropipette to introduce the solution directly or by using two capillaries placed through the walls of the PDMS chamber. The volume of microchambers was limited to $1 \mu \mathrm{L}$.

A concentrate aqueous solution of citrate $\mathrm{Au}$ particles of nominal diameter $100 \mathrm{~nm}$ was prepared by centrifugation of a colloidal solution (purchased from British Biocell International). Citrate ions adsorbed on the particle surface give the metallic colloïds a global negative charge, preventing their agglomeration in solution. In order to deposit particles on an insulating surface such as the $\mathrm{SiO}_{2}$ surface of our devices, we have chosen to perform a treatment using 3-aminopropyltrimethoxysilane (APTS) [7]. The grafting of this molecule on the surface leads to a polymerized film with amine groups. In solution, these groups provide to the surface a positive charge and the electrostatic interactions between nanoparticles and the silane treated surface are used to achieve their immobilization on the device [8]. A $10 \mathrm{mM}$ solution of APTS (purchased from ABCR) was prepared by dilution in a $95 \%$ ethanol $/ 5 \%$ water solution. After cleaning the substrate using ethanol with ultrasonic agitation followed by a plasma $\mathrm{O}_{2}$ treatment, samples were immersed in the APTS solution for $5 \mathrm{~min}$. After interaction, samples were rinsed in ethanol and baked for $20 \mathrm{~min}$ at $120^{\circ} \mathrm{C}$ in an oven. For grafting the $\mathrm{Au}$ particles onto the surface, a droplet of the colloidal solution was 


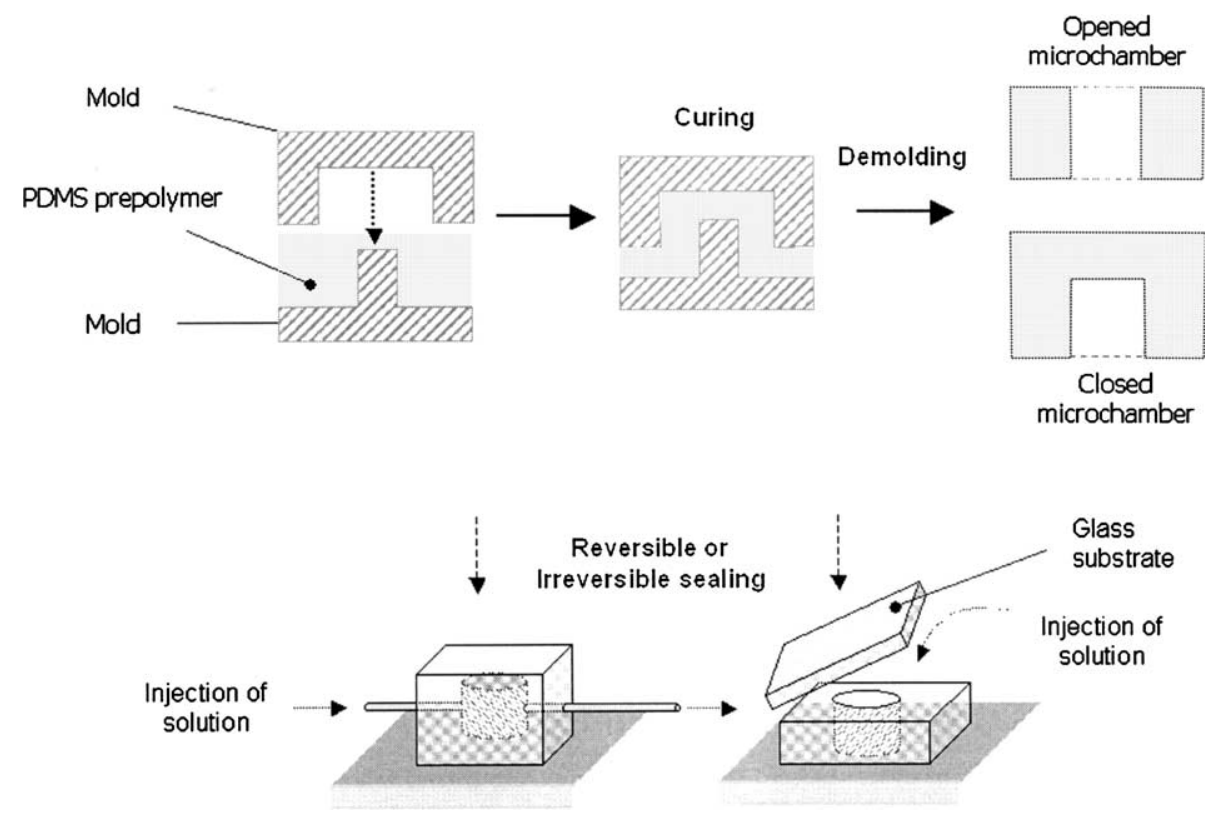

Fig. 2. Schematic description of the fabrication of PDMS microchambers dedicated to $I(V)$ measurements in solution.

directly deposited onto samples with a special attention to avoid evaporation. After interaction samples were washed with ethanol for $1 \mathrm{~min}$ and dried under nitrogen stream.

\section{Results and discussion}

First experiments were dedicated to the validation of the detection scheme. Using Gold nanoparticles in solution, we proceed to a deposition on interdigitated nanoelectrodes functionalized with APTS. The gap between electrodes was estimated at $60 \mathrm{~nm}$. A result of a SEM observation is reported in Fig. 3(a) and shows clearly the grafting of particles on the electrode array. The interaction time was chosen as $30 \mathrm{~min}$ to limit the particle density on the electrodes and to evaluate the sensitivity of our devices. The particle density was evaluated as 10 particles $\mu \mathrm{m}^{2}$. In this image it is possible to estimate the number of gold particles bridging two electrodes at 35. That gives an arrival rate of 1 particle/minute on the nanodevice. Conduction measurements through electrodes were carried out after colloid deposition and were compared to results obtained on untreated devices. In the absence of colloids, $I(V)$ character- istics indicate no measurable current even under a $10 \mathrm{~V}$ bias. No significant change of the device conductance was found until the chip was exposed to the colloidal solution. Fig. 3(b) shows an example of the $I(V)$ response of a typical device before and after colloidal interaction. As can be clearly seen, the interaction with colloids leads to a drastic change of the device conductivity. Under $1 \mathrm{~V}$ bias, the current level changes from $50 \mathrm{fA}$ to $120 \mathrm{pA}$. The resistance of the device around $1 \mathrm{~V}$ reaches a value of $2.5 \mathrm{G} \Omega$ after interaction, leading to a typical resistance of approximately $90 \mathrm{G} \Omega$ for a single particle. It is clear that the particles do not form an ohmic contact with the nanoelectrodes but tunnel junctions are formed between each particle and the metallic surface of the electrodes. Several experiments have confirmed these typical values and show that the electrical response changes accordingly to the number of particles immobilized on the nanodevice. These results confirm the compatibility of the architecture of these devices with colloids and the high sensitivity of this detection scheme.

The ability to perform in situ measurements during the incubation of gold colloids was also studied using a similar detection scheme. For this purpose, we used a moulded PDMS microchamber 

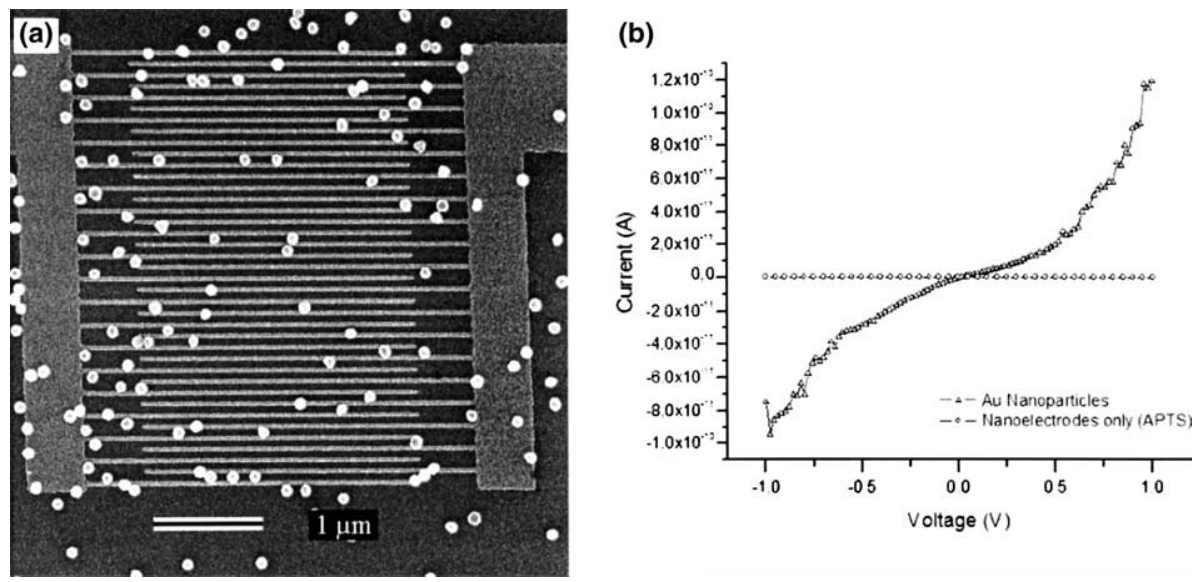

Fig. 3. (a) SEM image of an interdigitated nanoelectrode device after the grafting of $100 \mathrm{~nm}$ Au particles during 30 min and subsequent washing and drying. (b) $I(V)$ measurements show the electrical response of this nanodevice at room temperature before and after the deposition of the particles.

deposited on the surface of a interdigitated nanoelectrodes device with $60 \mathrm{~nm}$ gaps treated with APTS. A droplet of $100 \mathrm{~nm}$ colloids solution was then introduced into the chamber and a bias of $1 \mathrm{~V}$ applied to electrodes. Fig. 4(a) shows a typical evolution of the current during the incubation in the solution. Reproducible abrupt current changes were observed with amplitudes varying from 10 to $50 \mathrm{pA}$. These sudden modifications of the current can be attributed to the creation of new conduction paths as deposit progresses corresponding to a single binding event. Indeed, as seen previously the typical resistance at room temperature of a single colloid is $90 \mathrm{G} \Omega$, under a bias of $1 \mathrm{~V}$ this gives a current of $11 \mathrm{pA}$ which corresponds well with the typical jumps observed in Fig. 4(a). We thus think that each jump is the signature of a single colloid detection. Observations and measurements after rinsing and drying the sample confirmed the grafting of colloids between electrodes but it is not possible to correlate easily the number of current jumps with the number of bridging particles for two reasons. The first one is due to technical limitations due to the prober equipment which pre-
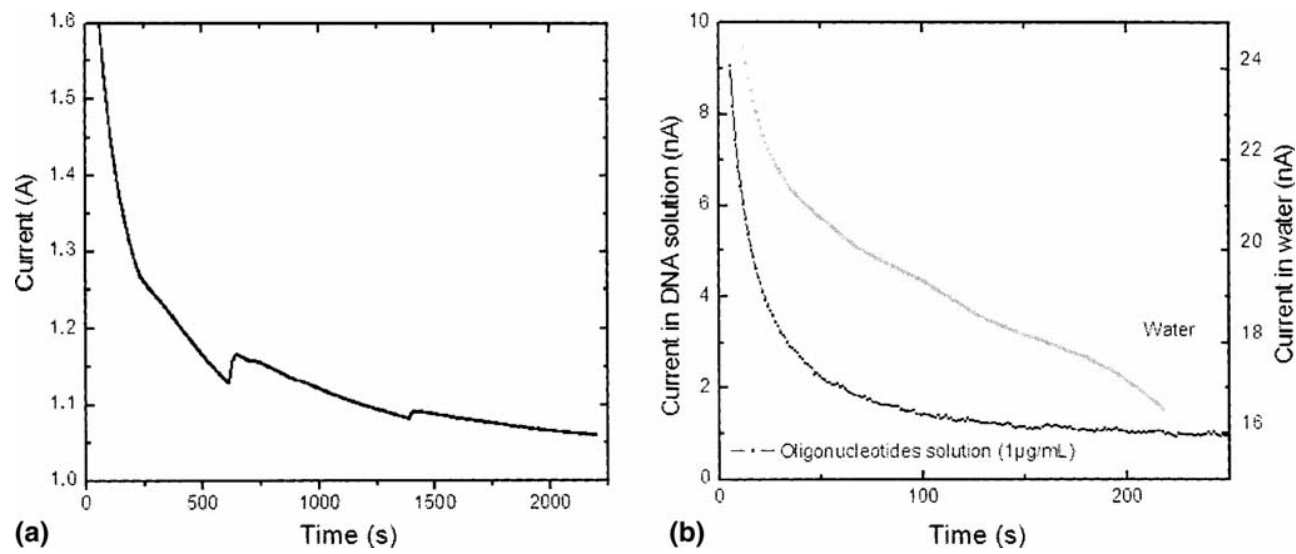

Fig. 4. Current dependence on time for an interdigitated nanodevice with $1 \mathrm{~V}$ bias applied: (a) during the incubation of a colloidal solution, the reproducible jumps of current are attributed to single particle detection; (b) during the incubation of an oligonucleotide solution in DI water. For comparison, the same curve obtained with pure DI water is displayed. Note the increase noise level observed with the DNA solution. 
vent the use of our injection system when the sample is loaded. As a consequence, no electrical measurement was possible during a transient phase after liquid injection. Improvements of the compatibility of the two equipments are in progress. Secondly, it is not yet clear if each trapping event give rise to a characteristic jump.

Experiments with unlabelled oligonucleotides were also carried out using a similar approach. A solution of $100 \mathrm{~nm}$ long $\lambda$-DNA molecules diluted in deionized water at a concentration of $1 \mu \mathrm{g} / \mathrm{mL}$ was injected using our fluidic cell onto an array of $40 \mathrm{~nm}$ (line/space) interdigitated electrodes biased at $1 \mathrm{~V}$. In that case, we do not evidence any abrupt change of current (see Fig. 4(b)). The same experiment using pure DI water injection gives a similar $I(t)$ behaviour. The only slight difference is the visible noise level of the curve obtained with DNA molecules in the solution (see Fig. 4(b)). We conclude once again, that the conductivity level of long DNA molecules is not sufficient for enabling the detection of molecule trapping in solution. Further experiments are necessary for elucidating the origin of the increased noise in relation with the DNA molecules around the nanoelectrodes.

\section{Conclusion}

Nanoelectrode-based devices compatible with electrical measurements in solution have been produced and applied to the detection of $100 \mathrm{~nm}$ $\mathrm{Au}$ particles. We demonstrate a high level of sensitivity. A small number of colloids can be detected after interaction and drying using $I(V)$ measurements and single-colloid detection seems to be possible in solution using $I(t)$ measurements. Next experiments will be dedicated to the detection of specific interactions between biomolecules labelled with $\mathrm{Au}$ colloids. This work opens the perspective of realizing bioassays with an integrated electrical detection using high sensitive nanodevices.

\section{References}

[1] R. Marie, J. Thaysen, C.B.V. Christensen, A. Boisen, Microelectron. Eng. 67-68 (2003) 893.

[2] A.J. Haes, R.P. Van Duyne, J. Am. Chem. Soc. 124 (2002) 10596.

[3] D. Porath, A. Berzyadin, S. de Vries, C. Dekker, Nature 403 (2000) 635.

[4] S.J. Park, T.A. Taton, C.A. Mirkin, Science 295 (2002) 503.

[5] A.J. Storm, J. van Noort, S. de Vries, C. Dekker, Appl. Phys. Lett. 79 (2001) 3881.

[6] Y. Xia, G.M. Whitesides, Annu. Rev. Mater. Sci. 28 (1998) 153-184.

[7] T. Sato, D.G. Hasko, H. Ahmed, J. Vac. Sci. Technol. B 15 (1997) 45.

[8] A. Shipway, E. Katz, I. Willner, Chem. Phys. Chem 1 (2000) 18. 